\title{
The Effects of Berberine on Blood Lipids: A Systemic Review and Meta-Analysis of Randomized Controlled Trials
}

Authors

Affiliations

\author{
Hui Dong ${ }^{1 *}$, Yan Zhao ${ }^{2}$, Li Zhao ${ }^{1 *}$, Fuer Lu
}

${ }^{1}$ Institute of Integrated Traditional Chinese and Western Medicine, Tongji Hospital, Tongji Medical College, Huazhong University of Science and Technology, Wuhan, Hubei, P. R. China

${ }^{2}$ Department of Integrated Traditional Chinese and Western Medicine, Tongji Hospital, Tongji Medical College, Huazhong University of Science and Technology, Wuhan, Hubei, P. R. China

Key words
berberine
lipid
systematic review
meta-analysis
randomized controlled trials
clinical trials

received June 3, 2012

revised January 31, 2013

accepted February 13, 2013

Bibliography

Dol http://dx.doi.org/

10.1055/s-0032-1328321

Published online March 19,

2013

Planta Med 2013; 79: 437-446

(c) Georg Thieme Verlag KG

Stuttgart · New York .

ISSN 0032-0943

\section{Correspondence \\ Dr. Hui Dong}

Institute of Integrated

Traditional Chinese and Western

Medicine

Tongji Hospital, Tongji Medical

College

Huazhong University of Science

and Technology

1095 Jiefang Avenue

Wuhan, Hubei 430030

People's Republic of China

Phone: + 862783663660

Fax: +862783663237

tjhdonghui@163.com

\section{Correspondence}

\section{Prof. Fuer Lu}

Institute of Integrated

Traditional Chinese and Western

Medicine

Tongji Hospital, Tongji Medical

College

Huazhong University of Science

and Technology

1095 Jiefang Avenue

Wuhan, Hubei 430030

People's Republic of China

Phone: + 862783663660

Fax: +862783663237

felu@tjh.tjmu.edu.cn

\section{Abstract}

\section{$\nabla$}

Clinical trials have reported lipid-lowering effects of berberine intake, but the findings have been inconsistent. The aim of this meta-analysis was to assess the safety of berberine and its effects on blood lipid profiles. A systemic review was designed, undertaken and reported in accordance with the PRISMA statement. Randomized controlled trials of the effects of berberine on blood lipids in adults were included. Study population characteristics and the main results, including changes in the levels of total cholesterol, triglycerides, low-density and high-density lipoprotein cholesterol, were extracted. Weighted mean differences were calculated for net changes in blood lipid concentrations using fixed-effect or random-effects models. After filtering, eleven randomized controlled trials (including a total of 874 participants) were included in this study. The methodological quality of these studies was generally low. The final analysis showed that administration of berberine produced a significant reduction in total cholesterol (mean difference $-0.61 \mathrm{mmol} / \mathrm{L} ; 95 \%$ confidence interval -0.83 to -0.39 ), triglycerides (mean difference

\section{Introduction}

\section{$\nabla$}

Hyperlipidemia is a condition characterized by the presence of excess fatty substances such as cholesterol and TG in the blood. A recent study using data from 1999 to 2006 showed that the unadjusted prevalence of hypercholesterolemia ranged from $53.2 \%$ to $56.1 \%$ in United States adults and changed little over the study period [1]. These results indicate that the hypercholesterolemia epidemic is a weighty problem and that

${ }^{*}$ Equal contribution.
$-0.50 \mathrm{mmol} / \mathrm{L} ; 95 \%$ confidence interval -0.69 to -0.31), and low-density lipoprotein cholesterol (mean difference $-0.65 \mathrm{mmol} / \mathrm{L}$; $95 \%$ confidence interval -0.76 to -0.54 ) levels, with a remarkable increase in high-density lipoprotein (mean difference $0.05 \mathrm{mmol} / \mathrm{L} ; 95 \%$ confidence interval 0.02 to 0.09 ). No serious adverse effects of berberine have been reported. In conclusion, berberine may have beneficial effects in the control of blood lipid levels. However, the efficacy of berberine in treating hyperlipidemia should be further evaluated by more randomized controlled trials in a larger population of patients.

\section{Abbreviations \\ $\nabla$ \\ $\mathrm{CI}$ : confidence interval \\ CYPs: $\quad$ cytochrome P450 enzymes \\ HDL-C: high-density lipoprotein cholesterol \\ LDL-C: low-density lipoprotein cholesterol \\ MD: $\quad$ weighted mean differences \\ RCTs: randomized controlled trials \\ TC: $\quad$ total cholesterol \\ TG: triglycerides}

control of hypercholesterolemia remains poor Generally, hyperlipidemia has no specific symptoms and is usually diagnosed during routine blood tests. However, elevated fatty substances may deposit in the form of plaques in the walls of arteries, a condition known as atherosclerosis, which leads to obstructions in blood flow [2]. These obstructions significantly increase the risk of experiencing a cerebral or myocardial infarction $[3,4]$.

The goal of treating hyperlipidemia is to lower the concentrations of LDL-C and TG and to raise the concentrations of HDL-C. Hyperlipidemia may be hereditary or caused by certain medications and/ 
or underlying medical problems such as diabetes and hypothyroidism. To control blood lipid concentrations, these conditions should be treated first. As a second line of treatment, dietary changes, weight loss, and exercise are recommended, followed by the use of statins, fibrates, or other lipid-lowering agents [58]. Evidence from many clinical trials has demonstrated that these prescription drugs can lower blood lipids and reduce the risk of developing cardiovascular diseases [3,9]. However, many patients experience adverse effects of these lipid-lowering drugs. For example, statins and fibrates are both linked to rhabdomyolysis and elevated liver enzyme activity, which can be life-threatening in some cases [10-12].

In China, herbal medicines have been commonly used as an alternative approach for treating hyperlipidemia [13]. Berberine, an isoquinoline derivative alkaloid isolated from several Chinese herbal medicines, such as Rhizoma Coptidis (Latin name: Coptis chinensis Franch, Ranunculaceae; Chinese name: Huang Lian), Amur Corktree Bark (Latin name: Cortex Phellodendri; Chinese name: Huang Bo), and Chinese Mahonia Stem (Latin name: Caulis Mahoniae; Chinese name: Gong Lao Mu), has been found to have beneficial effects on blood lipids [14]. A number of clinical trials have also been published on this subject in medical journals over the past 20 years. However, the findings have been inconsistent. Most studies have reported that berberine had beneficial effects on hyperlipidemia, while a few studies found that berberine had no effect on blood lipid concentrations [15]. It is also unclear if there is any therapeutic difference between the use of berberine alone and its use in combination with other lipid-lowering agents. The safety of long-term berberine intake in hyperlipidemic patients is also unknown. In this paper, we performed a meta-analysis of randomized controlled trials to systematically review the potential roles and long-term effects of berberine in the regulation of blood lipids.

\section{Materials and Methods}

$\nabla$

To ensure the rigor of our systemic review and meta-analysis, we designed and reported our findings using a checklist of items in accordance with the "Preferred Reporting Items for Systemic Review and Meta-Analyses (PRISMA)" statement as far as possible [16].

\section{Search strategy}

We searched the following electronic databases to identify trials: Medline, Embase, the Cochrane Library, the China Hospital Knowledge Database (CHKD), and the Wanfang database. Databases of ongoing trials were also searched. All the above databases were searched from the available date of inception until the latest issue (April 2012). No language restriction was used.

We combined different search strategies as follows: for the English databases (Medline and Embase), we used the MeSH terms and free text words "berberine" and "lipids" and "human"; for the Cochrane Library, we used the free text words "berberine" and "lipids"; for the Chinese databases (CHKD and Wanfang), we searched for the free text words "Huang Lian Su" or "Xiao Bo Jian" (which are both the alternative names of a single chemical entity of berberine in Chinese) and "Xue Zhi" or "Zhi Xue" (which means blood lipids in Chinese) in abstracts. A filter for clinical trials was applied. The reference lists of the included trials were also searched to identify additional studies.

\section{Selection criteria}

We included RCTs irrespective of blinding, publication status, or language. Studies were selected for analysis if they met the following criteria: 1) the subjects consumed a single chemical entity of berberine alone or with other pharmaceutical agents for at least 4 weeks; 2 ) the study was an RCT with either a parallel or a crossover design; 3 ) berberine was used as the active treatment intervention; and 4) the effects of berberine on blood lipid profiles (including TC, TG, LDL-C. and HDL-C) could be extracted from the report. There was no restriction on the type of disease that the trials aimed to study. However, no intervention difference was identified between the active treatment and control groups other than berberine intake. Therefore, a treatment group and a no berberine control group were included in each of the trials.

"Berberine", in this review, refers to the single chemical entity of berberine formulated as capsules or tablets. Therefore, trials using any berberine-containing herbs and herbal extracts were excluded. We also excluded case reports and studies without a control group. For obvious duplicate studies, authors of reports were contacted to clarify uncertainty. If the author could not be contacted, the first published report was regarded as the original. Studies were also excluded if the dose of berberine was not provided. RCTs without a clear description of blood lipid profiles, particularly those with insufficient data to allow calculation of the net change in blood lipids and their variances from baseline to the end of follow-up, were also excluded from our analysis. Two independent reviewers selected articles. A flow diagram of study selection was generated according to the PRISMA requirements.

\section{Data extraction and management}

Data were extracted independently by two reviewers, and any disagreements were resolved by consensus, or if necessary by a third reviewer. Study quality was independently assessed using the Jadad 5-point score plus allocation concealment for RCTs [17]. The Jadad score included method of randomization (0-2 points), double blinding ( $0-2$ points), and description of withdrawals or dropouts ( $0-1$ point). Allocation concealment was assessed by the criteria adopted from the Cochrane Handbook [18] and Schulz et al. [19]. Studies with a Jadad score of 3 or above were regarded as high quality.

We selected the following lipid biomarkers as outcomes: TC, TG, LDL-C, and HDL-C. Adverse effects were also pooled from the studies. In the trials which adopted a three- or four-armed group design, the outcomes of the groups were extracted when they met the inclusion criteria. Otherwise, the outcomes were discarded. In cases where the outcomes were ambiguous or missing in the article, the authors were contacted. If the authors could not be contacted, the decision to extract the data was resolved by consensus.

\section{Data synthesis and analysis}

The effects of berberine intake on blood lipids were calculated as differences between the treatment groups and the no berberine control group using Review Manager 5.1 meta-analysis software [20]. For parallel trials, the net change in blood lipids was calculated as the mean difference (berberine minus control) of the change (endpoint minus baseline) in blood lipids. Because the variances for net changes in blood lipids were not reported directly in most studies, they were calculated from $\mathrm{CI}$ for the berberine and control groups. We calculated MD and the $95 \% \mathrm{CI}$ for 


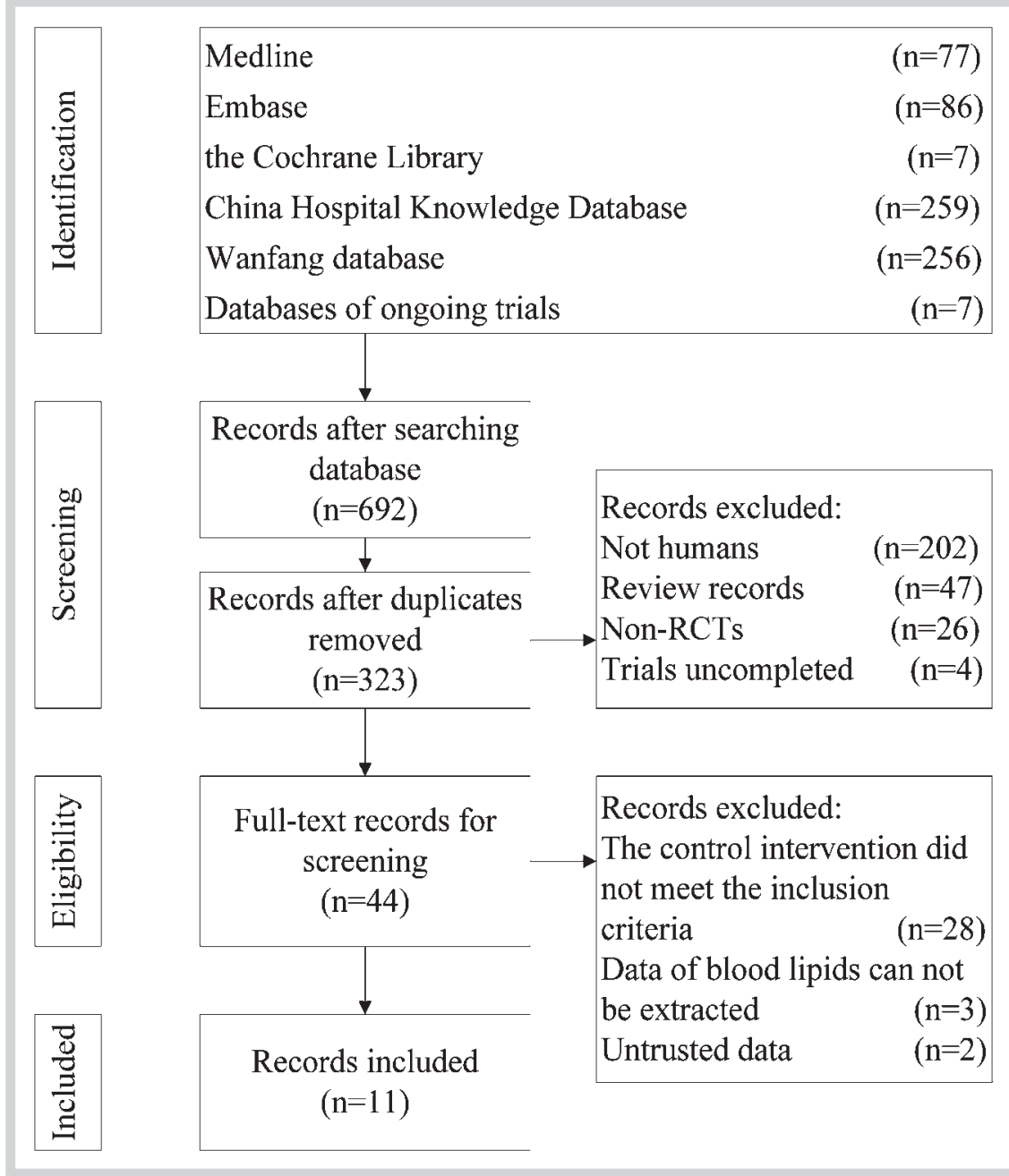

Fig. 1 Process of searching and screening studies.

continuous data. Heterogeneity was evaluated using the chisquare test, $\operatorname{tau}^{2}$ test, and the Higgins $I^{2}$ test. We presented the results based on a fixed-effect model when the studies in the group were sufficiently similar $(p>0.10)$. Otherwise, a random-effects model was used. The overall effect was tested using a Z score with significance set at $\mathrm{p}<0.05$. Funnel plots, Egger's regression asymmetry test, and Begg's test were used to detect publication bias using Stata 12.0 software. We conducted subgroup analyses to determine whether the use of berberine alone or with statins had different effects on lipid parameters. We also performed sensitivity analyses by repeating the analysis, this time taking into account the risk of bias and excluding any very long or large studies. Furthermore, we tested the robustness of the results by repeating the analysis using different statistical models.

\section{Results}

Study selection: The process of study selection is depicted in - Fig. 1. According to the prespecified selection criteria described in the Methods section, thirteen RCTs involving the effects of berberine on blood lipids were identified. Two trials [21,22] were excluded. One [21] of the included trials consisted of a threearm, parallel-group, randomized control trial to compare the effects of berberine, simvastatin, and the combination of both drugs on blood lipids in patients with hyperlipidemia. However, we excluded this trial based on untrustworthy data concerning the HDL-C concentration, which remained unchanged at $0,1,3$, and 6 months after simvastatin intake. We excluded another trial [22] based on the similarity of the TC and LDL-C data in the same group. Eleven RCTs met our inclusion criteria, and the characteristics of the trials are summarized in Table 1. Together, those trials included a total of 874 patients.

Description of the studies: The included studies were published as full text between 2004 and 2012. All RCTs originated from China. Four studies were published in English [14,23-25], and the remaining seven studies were published in Chinese [26-32]. Ten trials were performed as single center trials, while one study [24] was a multicenter trial. As shown in Table 1, four studies $[26,27,29,31]$ enrolled patients with type 2 diabetes. One study enrolled patients with type 2 diabetes and hyperlipidemia [24]. One study [28] enrolled patients with impaired glucose tolerance and hyperlipidemia. Two studies enrolled patients with hyperlipidemia [30,32]. Two studies [14,23] enrolled patients with hypercholesterolemia. One study [25] enrolled patients with polycystic ovary syndrome and insulin resistance.

Intervention and controls: Six studies randomized participants to receive berberine alone or with a co-intervention of lifestyle changes versus a control of placebo with or without lifestyle changes. Two trials compared a co-intervention of berberine and oral hypoglycemics (metformin, glipizide, or glimepiride) with a control of the same hypoglycemics. Two trials compared a co-in- 
Table 1 Characteristics of included trials.

\begin{tabular}{|c|c|c|c|c|c|c|c|}
\hline \multirow[t]{2}{*}{ Author } & \multirow{2}{*}{$\begin{array}{l}\text { Primary } \\
\text { diagnosis }\end{array}$} & \multicolumn{2}{|c|}{ Number of patients } & \multicolumn{2}{|c|}{ Intervention and Berberine dose } & \multirow{2}{*}{$\begin{array}{l}\text { Duration } \\
\text { (wks) }\end{array}$} & \multirow[t]{2}{*}{ Outcomes } \\
\hline & & Berberine & Control & Berberine & Control & & \\
\hline Kong 2004 [14] & $\mathrm{HC}$ & 63 & 28 & BBR (0.5 g twice daily) & PL & 12 & TC, TG, LDL-C, HDL-C, AE \\
\hline Li 2007 [26] & $\mathrm{DM}$ & 51 & 50 & $\begin{array}{l}\text { BBR ( } 0.3 \mathrm{~g} \text { three times } \\
\text { daily), GLIP }\end{array}$ & GLIP & 8 & TC, TG, LDL-C, HDL-C, AE \\
\hline Cao 2007 [27] & DM & 30 & 30 & $\begin{array}{l}\text { BBR ( } 0.5 \mathrm{~g} \text { three times } \\
\text { daily), } \mathrm{LM}\end{array}$ & LM & 12 & TC, TG, LDL-C, HDL-C, AE \\
\hline Ju 2007 [28] & HL,IGT & 45 & 42 & $\begin{array}{l}\text { BBR ( } 0.2 \mathrm{~g} \text { three times } \\
\text { daily), LM }\end{array}$ & LM & 52 & TC, TG, LDL-C, HDL-C, AE \\
\hline Wang 2008 [29] & DM & 30 & 31 & $\begin{array}{l}\text { BBR ( } 0.3 \mathrm{~g} \text { three times } \\
\text { daily), LM }\end{array}$ & LM & 12 & TC, TG, LDL-C, HDL-C, AE \\
\hline Kong 2008 [23] & $\mathrm{HC}$ & 23 & 16 & $\begin{array}{l}\text { BBR ( } 0.5 \mathrm{~g} \text { twice dai- } \\
\text { ly), SIM }\end{array}$ & SIM & 8 & TC, TC, LDL-C, HDL-C, AE \\
\hline $\begin{array}{l}\text { Zhang } 2008 \\
\text { [24] }\end{array}$ & $\mathrm{DM}, \mathrm{HL}$ & 58 & 52 & $\begin{array}{l}\text { BBR ( } 0.5 \text { g twice dai- } \\
\text { ly), LM }\end{array}$ & PL, LM & 12 & TC, TG, LDL-C, HDL-C, AE \\
\hline $\begin{array}{l}\text { Zheng } 2009 \\
{[30]}\end{array}$ & $\mathrm{HL}$ & 33 & 33 & $\begin{array}{l}\text { BBR }(0.4 \mathrm{~g} \text { three times } \\
\text { daily), SIM }\end{array}$ & SIM & 8 & TC, TG, LDL-C, HDL-C, AE \\
\hline Ye 2010 [31] & DM & 40 & 40 & $\begin{array}{l}\text { BBR ( } 0.5 \mathrm{~g} \text { three times } \\
\text { daily), MET, GLIM }\end{array}$ & MET, GLIM & 12 & TC, TG, LDL-C, HDL-C, AE \\
\hline $\begin{array}{l}\text { Zhou } 2011 \\
\text { [32] }\end{array}$ & $\mathrm{HL}$ & 60 & 60 & $\begin{array}{l}\text { BBR ( } 0.3 \mathrm{~g} \text { three times } \\
\text { daily), } \mathrm{LM}\end{array}$ & LM & 16 & TC, TG, LDL-C, HDL-C \\
\hline Wei 2012 [25] & PCOS,IR & 31 & 28 & $\begin{array}{l}\text { BBR }(0.5 \mathrm{~g} \text { three times } \\
\text { daily), CPA, LM }\end{array}$ & PL, CPA, LM & 12 & TC, TG, LDL-C, HDL-C, AE \\
\hline
\end{tabular}

Note: AE, adverse effect; BBR, berberine; CPA, cyproterone acetate; DM, type 2 diabetes mellitus; GLIM, glimepiride; GLIP, glipizide; HC, hypercholesterolemia; HL, hyperlipidemia; IGT, impaired glucose tolerance; IR, insulin resistance; LM, lifestyle modification; MET, metformin; PCOS, polycystic ovary syndrome; PL, placebo; SIM, simvastatin

tervention of berberine and simvastatin with a control of simvastatin. One trial compared a co-intervention of berberine and cyproterone acetate with a control of placebo and cyproterone acetate.

There were two types of berberine formulation used in the included trials, consisting of berberine chloride tablets (used in ten trials) and berberine chloride liposome capsules (used in only one trial). Different doses of berberine were used in these trials. Berberine intake was generally in the range of $0.5 \mathrm{~g}$ to $1.5 \mathrm{~g}$ per day. The total daily berberine intake was divided into two or three doses. The dose of berberine was unchanged during the period of study in eight trials. Three trials $[14,24,29]$ reduced the dose of berberine during the period of study when gastrointestinal discomfort occurred.

The duration of interventions in the trials included also differed among the studies and ranged from eight to fifty-two weeks. The interventions lasted for eight weeks or two months in three trials $[10,26,30]$ and twelve weeks or three months in six trials $[14,24$, $25,27,29,31]$. One trial [32] lasted for four months. Only one trial [28] reported that the patients had received intervention for one year.

Objectives and outcomes: All the included trials were performed to evaluate anti-dyslipidemic and/or hypoglycemic effects of berberine. The outcomes reported were mainly surrogate parameters, including blood lipids and/or glucose levels. Adverse effects were reported in ten trials. All the reported outcomes were measured from the baseline to the end of the intervention. Ten trials performed treated-per-protocol analysis and one [24] performed intention-to-treat analysis.

Quality of the included studies: Most of the included trials in this meta-analysis were of poor quality (Jadad score $<3$ ), suggesting a high risk of bias. Only two trials $[24,25]$ were of high quality, as indicated by adequate random sequence generation, adequate allocation concealment, and described withdrawal or dropouts. An adequate double blind design was also performed in one of these two trials [24].

Publication bias: Visual inspection of the funnel plot showed that there were no significant publication biases for the comparisons of TC, TG LDL-C, and HDL-C (data not shown). We also used Egger's linear regression test to assess publication bias statistically. As shown in 0 Fig. 2, 95\% confidence intervals for the intercept included zero, and the calculated p values exceeded 0.05 for all the comparisons among the studies (TC, $\mathrm{p}=0.077$; $\mathrm{TG}, \mathrm{p}=0.790$; LDL-C, $\mathrm{p}=0.097$; HDL-C, $\mathrm{p}=0.443$ ). Therefore, no publication bias was detected in the included studies.

The effect of berberine on blood lipid levels: Pooled estimates of the effects of treatment on the blood lipid concentrations are summarized in 0 Fig. 3. The statistical heterogeneity among studies was found to be significant with respect to the results for blood TC and TG $(p<0.10)$. The pooled results showed a significant difference between the berberine-treated group and the control group. Berberine was significantly better than the control in terms of improving blood TC $(\mathrm{p}<0.00001$; MD $-0.61 \mathrm{mmol} / \mathrm{L}$; $95 \% \mathrm{CI}-0.83$ to -0.39$)$ and TG ( $<<0.00001 ; \mathrm{MD}-0.50 \mathrm{mmol} / \mathrm{L}$; $95 \% \mathrm{CI}-0.69$ to -0.31$)$. There was no statistical heterogeneity among trials with respect to LDL-C and HDL-C ( $p>0.10)$. Compared with the control group, those taking berberine showed significantly better results for LDL-C $(p<0.00001$; MD $-0.65 \mathrm{mmol} /$ $\mathrm{L} ; 95 \% \mathrm{CI}-0.76$ to -0.54$)$ and HDL-C ( $=0.001$; $\mathrm{MD} 0.05 \mathrm{mmol} / \mathrm{L}$; $95 \%$ CI 0.02 to 0.09 ).

Subgroup and sensitivity analyses: To better explain the effect of berberine on blood lipids, we performed two subgroup analyses. The first subgroup was the use of berberine alone or combined with lifestyle modification versus the control group. The second subgroup was the coadministration of berberine and simvastatin versus the control of simvastatin alone. Therefore, six trials [14, $24,27-29,32$ ] were included in the first subgroup, and two trials $[23,30]$ were included in the second subgroup. 

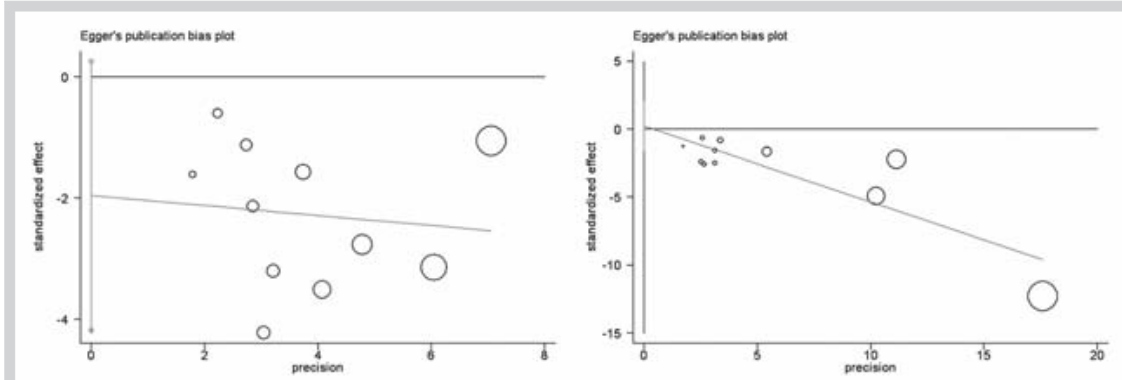

Fig. 2 Publication bias in the included trials. Egger's linear regression test for detecting publication bias. Note: TG, triglycerides; TC, total cholesterol; LDL-C, low-density lipoprotein cholesterol; HDL-C, high-density lipoprotein cholesterol. "O" is a size graph symbol by weights for each included study.
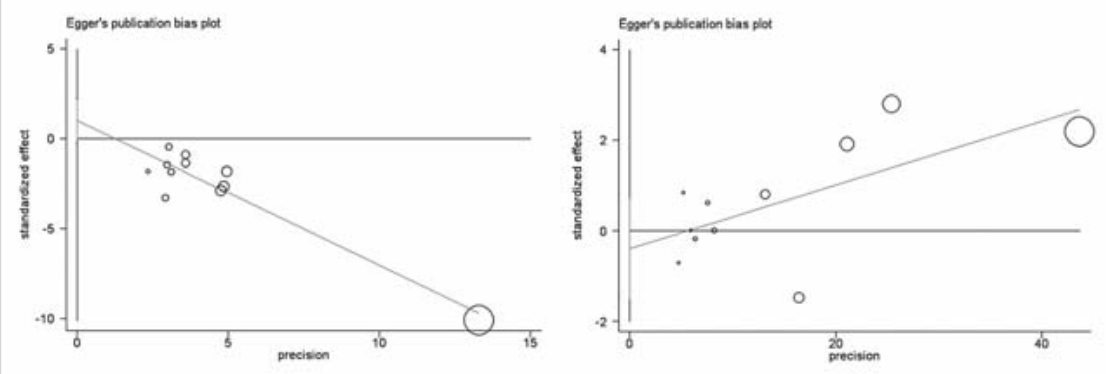

As shown in $\odot$ Fig. 4, berberine has a better efficacy than the control in terms of lowering blood TC ( $\mathrm{p}=0.0010$; MD $-0.56 \mathrm{mmol} /$ $\mathrm{L} ; 95 \% \mathrm{CI}-0.89$ to -0.23$)$, TG ( $\mathrm{p}<0.00001$; $\mathrm{MD}-0.67 \mathrm{mmol} / \mathrm{L}$; $95 \% \mathrm{CI}-0.78$ to -0.57$)$, LDL-C ( $<<0.00001$; MD $-0.68 \mathrm{mmol} / \mathrm{L}$; $95 \% \mathrm{CI}-0.80$ to -0.56$)$, and increasing HDL-C ( $\mathrm{p}=0.004$; MD $0.06 \mathrm{mmol} / \mathrm{L} ; 95 \% \mathrm{CI} 0.02$ to 0.09 ). Significant statistical heterogeneity was only identified in the TC endpoint analysis $(\mathrm{p}<0.10)$. As shown in $\odot$ Fig. 5 , adding berberine to simvastatin therapy significantly lowered blood TC $(p=0.02 ; \quad M D$ $-0.95 \mathrm{mmol} / \mathrm{L} ; 95 \% \mathrm{CI}-1.74$ to -0.16$)$, TG ( $\mathrm{p}=0.02 ; \mathrm{MD}$ $-0.58 \mathrm{mmol} / \mathrm{L} ; 95 \% \mathrm{CI}-1.06$ to -0.09 ), and LDL-C ( $\mathrm{p}=0.02$; MD $-0.61 \mathrm{mmol} / \mathrm{L} ; 95 \% \mathrm{CI}-1.13$ to -0.09 ). However, no effect on HDL-C ( $\mathrm{p}=0.56$; MD $-0.07 \mathrm{mmol} / \mathrm{L} ; 95 \% \mathrm{CI}-0.32$ to 0.17 ) was found with coadministration of berberine and simvastatin compared with administration of simvastatin alone. Significant statistical heterogeneity was also observed in the TC endpoint analysis $(\mathrm{p}<0.10)$.

No noteworthy changes in any of the study endpoints were noted after conducting sensitivity analyses.

Adverse effects: Ten trials reported outcomes for adverse effects. Eight trials $[14,23,24,26,27,29-31]$ stated that they asked the patients directly about the adverse effects while two trials [25, 28 ] did not state this. In three of these trials $[23,27,30]$, no adverse effects were reported during the berberine treatment. The rest of the trials mentioned in detail that adverse effects occurred in the berberine intervention group. Four trials [14,24, 29,31] reported mild to moderate constipation in a few participants who received berberine that was, however, relieved with or without dose reduction. One trial [26] reported that a few patients developed mild diarrhea caused by the intake of berberine, but they did not describe the population data in detail. Another trial [28] reported one case of abdominal distention after berberine intake. Finally, yet another trial [25] reported that three subjects who received berberine complained of a bitter taste in their mouth. No other side effects were observed in the included trials. There was also no significant difference between the berberine and the control groups regarding the incidence of adverse effects.

\section{Discussion}

In traditional Chinese medicine, hyperlipidemia falls into the category of symptoms caused by excess dampness. Increasing evidence indicates that berberine-containing herbs that remove accumulated pathogenic dampness are useful in treating hyperlipidemia $[33,34]$. These berberine-containing herbs are usually prescribed with other herbs and should always be administered as decoctions. For the variance in herbal prescription and the poor compliance of the patients (they should make an herbal decoction every day), the quality of clinical trials is difficult to be guaranteed. In contrast, isolated berberine, in the form of tablets and capsules, assures the quality control in clinical trials. In China, doctors often recommend tablets and capsules of herbal extracts for the convenience of ingestion. This is the reason why isolated berberine is selected in our meta-analysis. However, the difference in dampness removing or hypolipidemic effects between berberine-containing herbs and isolated berberine has rarely been reported.

As far as we know, our meta-analysis is the first quantitative review of randomized clinical trials yielding information on the effects of berberine on blood lipids. Eleven RCTs with a total of 874 Chinese patients were included. Participants were mainly patients with hyperlipidemia, type 2 diabetes mellitus, or both diseases. The results showed that berberine intake was associated with a significant decrease in blood TC $(0.61 \mathrm{mmol} / \mathrm{L}), \mathrm{TG}$ $(0.50 \mathrm{mmol} / \mathrm{L})$, and $\mathrm{LDL}-\mathrm{C}(0.65 \mathrm{mmol} / \mathrm{L})$ concentrations compared with the control group. A small but significant increase in blood HDL-C $(0.05 \mathrm{mmol} / \mathrm{L})$ concentration after berberine treatment was also found. This beneficial effect did not change when sensitivity analyses were performed. Subgroup analyses also yielded no changes in our conclusions, except for those concerning HDL-C. However, we should be cautious in interpreting this inconsistency in the HDL-C findings due to the limited number of trials included. Overall, our review indicates that berberine is effective at controlling blood lipid levels.

According to the NCEP ATP III 2004 update [35], LDL-C is the primary target of lipid-lowering therapy. The ATP III treatment algorithm also established different goals for LDL-lowering therapy based on the risk categories of the individual patient. Unfortu- 


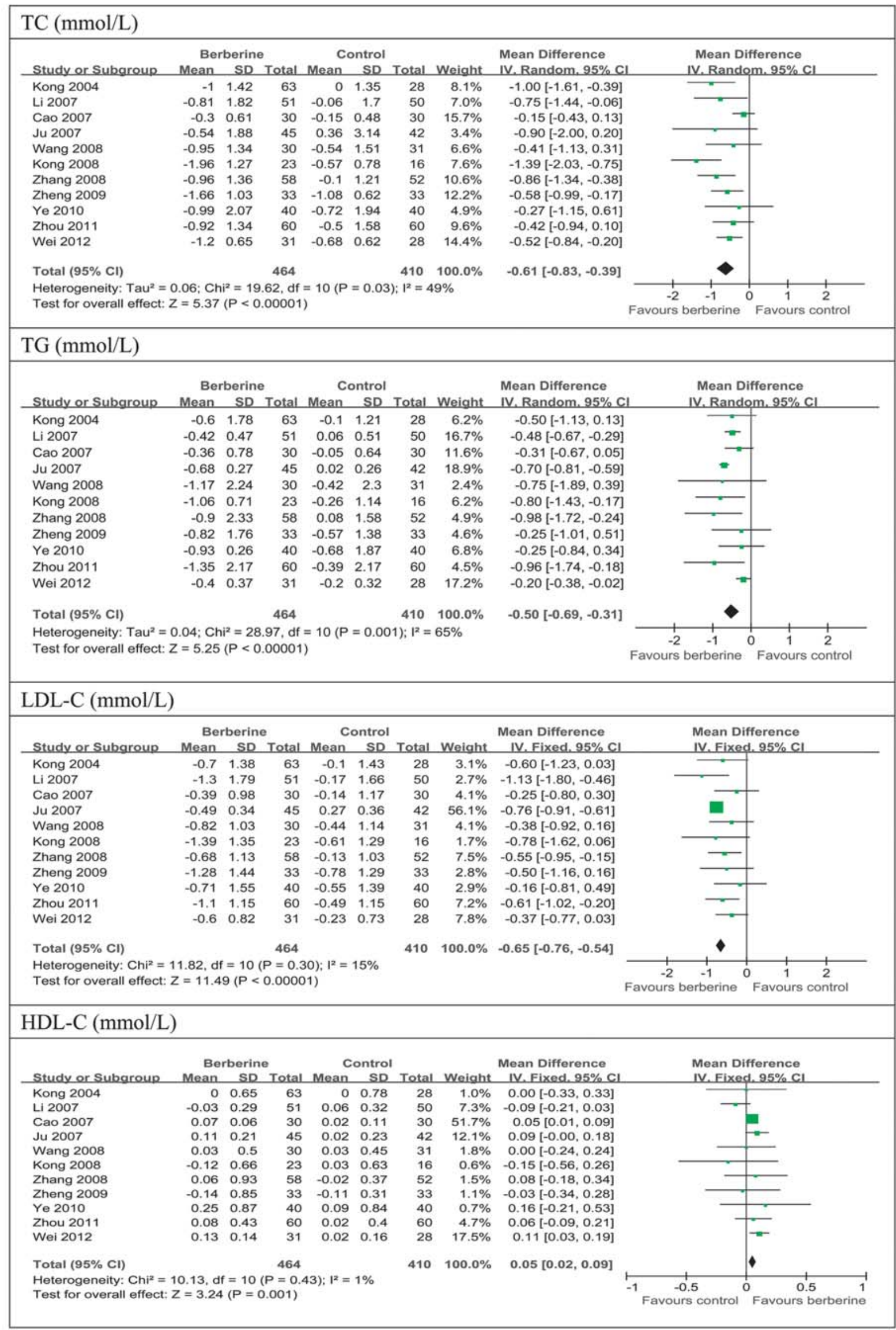

Fig. 3 Meta-analyses of the effects of berberine on blood lipids. Forest plots of berberine treatment versus control. Note: TG, triglycerides; TC, total cho- lesterol; LDL-C, low-density lipoprotein cholesterol; HDL-C, high-density lipoprotein cholesterol. (Color figure available online only.) 
nately, the information about these risk categories that was included in the trials we examined was insufficient; thus, we do not know whether the patients receiving berberine therapy reached the LDL-C goals. However, a large number of RCTs have demonstrated that effective LDL-C reduction reduces the risk for coronary heart disease. For every $30 \mathrm{mg} / \mathrm{dL}$ or $0.78 \mathrm{mmol} / \mathrm{L}$ change in LDL-C, the relative risk for coronary heart disease decreases by approximately $30 \%$ [35]. Thus, the $25 \mathrm{mg} / \mathrm{dL}$ $(0.65 \mathrm{mmol} / \mathrm{L}) \mathrm{LDL}-\mathrm{C}$ reduction observed in our meta-analysis is not only statistically significant but also likely to be clinically important. Similar LDL-C reductions were also found in subgroup analyses when berberine was used either alone (yielding a $26 \mathrm{mg} / \mathrm{dL}$ or $0.67 \mathrm{mmol} / \mathrm{L} \mathrm{LDL}-\mathrm{C}$ reduction) or added to statin therapy (yielding a $24 \mathrm{mg} / \mathrm{dL}$ or $0.61 \mathrm{mmol} / \mathrm{L}$ LDL-C reduction). These observations may assist us to draw a more solid conclusion about the efficacy of berberine in blood lipid control.

Herb-drug interactions might be a topic of concern if we recommend a co-intervention with berberine and statins for the treatment of hyperlipidemia. In recent years, the interactions between berberine/berberine-containing products and CYPs have been extensively reported. The results indicated that, at commonly recommended doses, berberine does not induce or inhibit the activity or gene expression of the CYPs. High doses of berberine may inhibit some types of CYPs, such as CYP3A11 and CYP3A25 [36,37]. Repeated oral administration of berberine $(0.3 \mathrm{~g}$ three times daily) decreased CYP2D6, CYP2C9, and CYP3A4 activities in healthy subjects [38]. Because the doses of berberine used in the studies included here were similar to or even higher than this dose, herb-drug interactions should be considered.

Although berberine generally appeared to be safe in the studies included here, a few side effects occurred. Gastrointestinal problems such as constipation, diarrhea, nausea, and abdominal distension were the most commonly reported side effects. After berberine intake, constipation was one of the most frequent gastrointestinal complaints; berberine has long been used to treat infectious diarrhea in China. However, these side effects were tolerable and were relieved after reducing the dose of berberine. No other serious adverse events were reported in the trials that reported safety data. However, the safety of using berberine in hyperlipidemic patients requires further evaluation, as the number of the trials included was limited.

Some limitations of this meta-analysis should be noted. First, all the participants were recruited from Chinese populations, indicating a risk of selection bias. This could affect the applicability of the interventions to populations from other ethnic origin. Second, three trials $[14,23,30]$ originated from the same group of investigators, suggesting a lack of independence in authorship. Although the authors, upon being contacted, declared that the trial participants were different in all of these studies, the inclusion of trials from the same authors has a high risk of bias. Third, most of the included studies were of poor quality in terms of their design, reporting, and methodology. We only identified one multicenter RCT [24] that used a double blind, adequate randomization and allocation concealment in the trials included. Nine of the trials included did not use blinding and allocation concealment completely. As we know, if participants are not blinded, knowledge of group assignment may affect responses to the intervention [39]. Furthermore, inadequate allocation concealment may lead to exaggerated estimates of treatment effect [40]. Fourth, there was a considerable heterogeneity among the trials, particularly with respect to TC $(p=0.03)$ and TG $(p=0.001)$. We think that differences in the participant charac- teristics, intervention methods, and treatment durations may have contributed to this heterogeneity. Taking this into consideration, the conclusions in our review should be carefully interpreted due to the substantial methodological and clinical diversity of the studies under review.

In summary, berberine may have beneficial effects on blood lipid control in clinical practice. Based on the existing limited evidence, berberine appears to have a favorable safety profile, but further investigation is required. Given the generally low methodological quality of the randomized trials, we suggest that more RCTs are needed if berberine is to be recommended for clinical use in the treatment of hyperlipidemia. Additionally, because reducing cardiovascular risk factors is the major focus of treating hyperlipidemia, further studies are required to investigate the effectiveness of berberine on cardiovascular risk factors other than blood lipids.

\section{Acknowledgements \\ $\nabla$}

The authors would like to thank Dr. Jia Lu for her help in improving the English of this paper and Prof. Liang Wang for the first comments on this paper. A grant from the National Natural Science Foundation of China (30801492) is also acknowledged.

\section{Conflict of Interest}

$\nabla$

We have no conflicts of interest to declare.

\section{References}

1 Ford ES, Li C, Pearson WS, Zhao G, Mokdad AH. Trends in hypercholesterolemia, treatment and control among United States adults. Int J Cardiol 2010; 140: 226-235

2 Ross R, Harker L. Hyperlipidemia and atherosclerosis. Science 1976; 193: $1094-1100$

3 Wang YR, Alexander GC, Meltzer DO. Screening and treatment of hypercholesterolemia for prevention of coronary heart disease. Manag Care Interface 2006; 19: 25-29

4 Lewis A, Segal A. Hyperlipidemia and primary prevention of stroke: does risk factor identification and reduction really work? Curr Atheroscler Rep 2010; 12: 225-229

5 Iughetti L, Bruzzi P, Predieri B. Evaluation and management of hyperlipidemia in children and adolescents. Curr Opin Pediatr 2010; 22: 485493

6 Executive summary of the third report of the National Cholesterol Education Program (NCEP) expert panel on detection, evaluation, and treatment of high blood cholesterol in adults (Adult Treatment Panel III). JAMA 2001; 285: 2486-2497

7 Staels B, Schoonjans K, Fruchart JC, Auwerx J. The effects of fibrates and thiazolidinediones on plasma triglyceride metabolism are mediated by distinct peroxisome proliferator activated receptors (PPARs). Biochimie 1997; 79: 95-99

8 Fruchart JC, Brewer Jr. HB, Leitersdorf E. Consensus for the use of fibrates in the treatment of dyslipoproteinemia and coronary heart disease. Fibrate Consensus Group. Am J Cardiol 1998; 81: 912-917

9 Abourbih S, Filion KB, Joseph L, Schiffrin EL, Rinfret S, Poirier P, Pilote L, Genest J, Eisenberg MJ. Effect of fibrates on lipid profiles and cardiovascular outcomes: a systematic review. Am J Med 2009; 122: 962 e1-e8

10 de Denus S, Spinler SA, Miller K, Peterson AM. Statins and liver toxicity: a meta-analysis. Pharmacotherapy 2004; 24: 584-591

11 Bates ER. Review: limited evidence suggests that statins are safe in patients with liver disease. Evid Based Med 2008; 13: 140

12 Matzno S, Tazuya-Murayama K, Tanaka H, Yasuda S, Mishima M, Uchida T, Nakabayashi T, Matsuyama K. Evaluation of the synergistic adverse effects of concomitant therapy with statins and fibrates on rhabdomyolysis. J Pharm Pharmacol 2003; 55: 795-802 


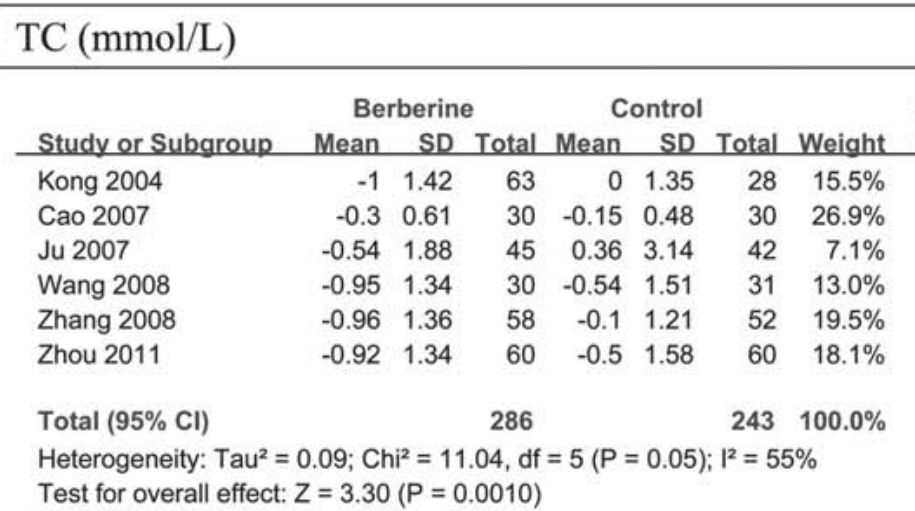

Test for overall effect: $Z=3.30(P=0.0010)$

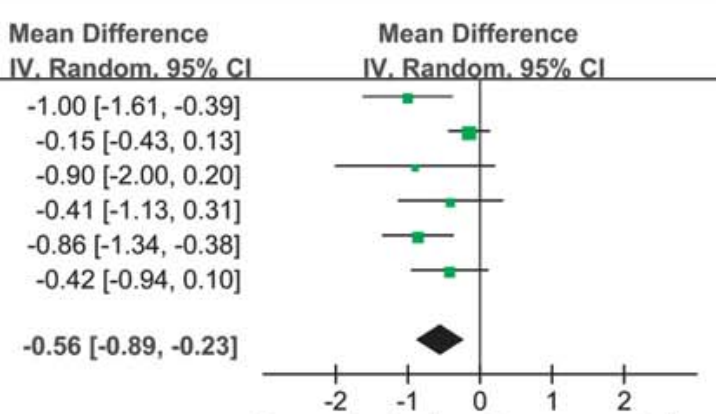

Favours berberine Favours control

\section{$\mathrm{TG}(\mathrm{mmol} / \mathrm{L})$}

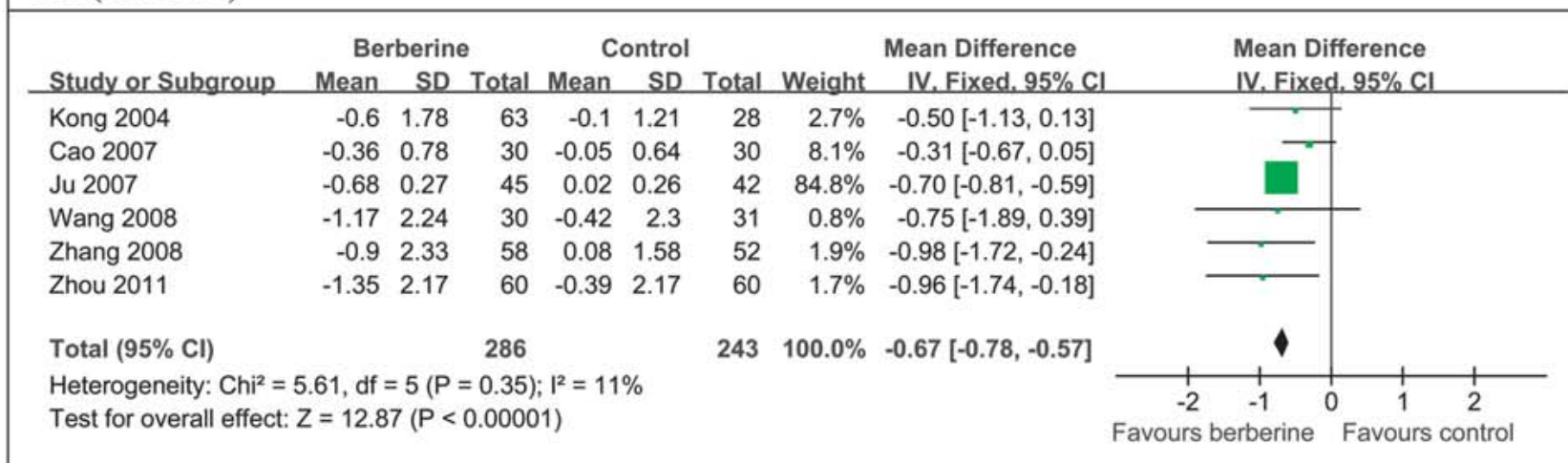

\section{LDL-C $(\mathrm{mmol} / \mathrm{L})$}

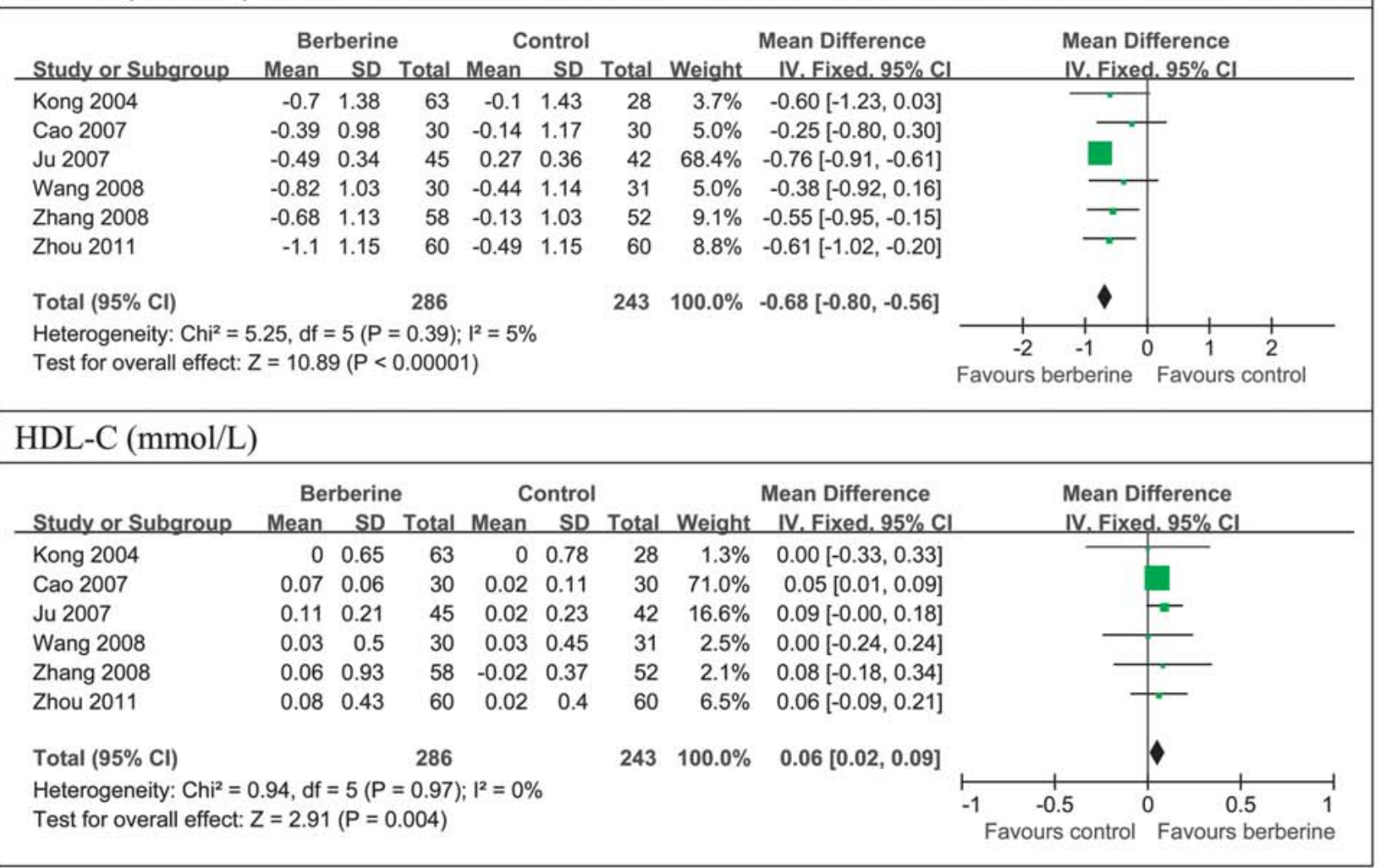

Fig. 4 The first subgroup analyses of the effects of berberine on blood lipids. Forest plots of the use of berberine alone or combined with lifestyle modification versus the control group. Note: TG, triglycerides; TC, total cho- lesterol; LDL-C, low-density lipoprotein cholesterol; HDL-C, high-density lipoprotein cholesterol. (Color figure available online only.) 


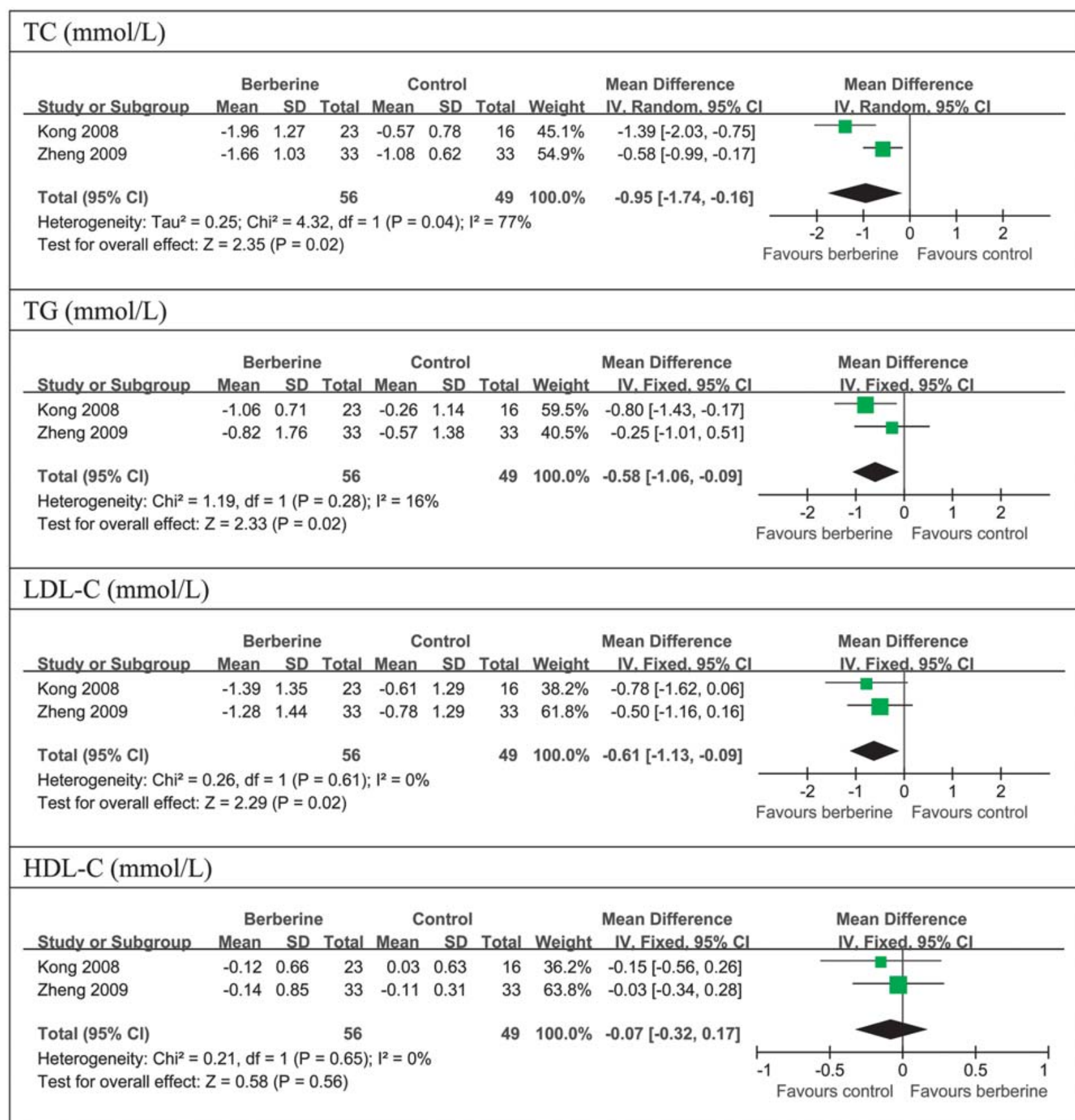

Fig. 5 The second subgroup analyses of the effects of berberine on blood lipids. Forest plots of the coadministration of berberine and simvastatin versus the control (simvastatin alone). Note: TG, triglycerides; TC, total choles- terol; LDL-C, low-density lipoprotein cholesterol; HDL-C, high-density lipoprotein cholesterol. (Color figure available online only.)
13 Liu ZL, Liu JP, Zhang AL, Wu Q, Ruan Y, Lewith G, Visconte D. Chinese herbal medicines for hypercholesterolemia. Cochrane Database Syst Rev 2011: CD008305

14 Kong W, Wei J, Abidi P, Lin M, Inaba S, Li C, Wang Y, Wang Z, Si S, Pan H, Wang S, Wu J, Wang Y, Li Z, Liu J, Jiang JD. Berberine is a novel cholesterol-lowering drug working through a unique mechanism distinct from statins. Nat Med 2004; 10: 1344-1351

15 Huang XS, Yang GF, Pan YC. Effect of berberine hydrochloride on blood concentration of cyclosporine $\mathrm{A}$ in cardiac transplanted recipients. Chin J Int Tradit West Med 2008; 28: 702-704
16 Moher D, Liberati A, Tetzlaff J, Altman DG; PRISMA Group. Preferred reporting items for systematic reviews and meta-analyses: the PRISMA statement. PLoS Med 2009; 6: e1000097

17 Jadad AR, Moore RA, Carroll D, Jenkinson C, Reynolds DJ, Gavaghan DJ, McQuay HJ. Assessing the quality of reports of randomized clinical trials: is blinding necessary? Control Clin Trials 1996; 17: 1-12

18 Higgins JPT, Green S. Cochrane Handbook for Systematic Reviews of Interventions, Version 5.0.0 [updated February 2008]. Chichester: The Cochrane Collaboration; 2008

19 Schulz KF, Chalmers I, Hayes RJ, Altman DG. Empirical evidence of bias: dimensions of methodological quality associated with estimates of treatment effects in controlled trials. JAMA 1995; 273: 408-412 
20 The Nordic Cochrane Centre. The Cochrane Collaboration, Review Manager (RevMan). 5.0. Copenhagen: The Nordic Cochrane Centre, The Cochrane Collaboration; 2008

$21 \mathrm{He} J \mathrm{~J}, \mathrm{Xu} X \mathrm{XG}$, Zheng $\mathrm{S}$. Therapeutic effects of simvastatin and berberine hydrochloride in the treatment of hyperlipidemia and carotid artery arthrosclerosis. Tianjin Pharm 2007; 35: 539-541

22 Yin SL, Li CH, Liu CM. The effects of berberine hydrochloride on blood glucose, insulin and lipids concentrations in newly diagnosed type 2 diabetic patients. Acta Acad Med Xuzhou 2011; 31: 40-41

23 Kong WJ, Wei J, Zuo ZY, Wang YM, Song DQ You XF, Zhao LX, Pan HN, Jiang JD. Combination of simvastatin with berberine improves the lipid-lowering efficacy. Metabolism 2008; 57: 1029-1037

24 Zhang Y, Li X, Zou D, Liu W, Yang J, Zhu N, Huo L, Wang M, Hong J, Wu P, Ren G, Ning G. Treatment of type 2 diabetes and dyslipidemia with the natural plant alkaloid berberine. J Clin Endocrinol Metab 2008; 93: 2559-2565

25 Wei W, Zhao H, Wang A, Sui M, Liang K, Deng H, Ma Y, Zhang Y, Zhang H, Guan Y. A clinical study on the short-term effect of berberine in comparison to metformin on the metabolic characteristics of women with polycystic ovary syndrome. Eur J Endocrinol 2012; 166: 99-105

$26 \mathrm{Li} \mathrm{Z}$, Liu $\mathrm{LH}$. Therapeutic efficacy of combined berberine and glipizide on type 2 diabetes. J Clin Res 2007; 24: 61-64

27 Cao Y. Clinical research on multiple factor intervention of improving the insulin resistance. Jinan: Shandong University of Traditional Chinese Medicine; 2007

$28 \mathrm{Ju} S B$, Tan $L R$, Su W, Rong $K W$. The intervention of berberine hydrochloride liposomes on impaired glucose tolerance combining with hyperlipidemia. J Pract Tradit Chin Med 2007; 23: 490-491

29 Wang $W$. Effect of berberine on blood glucose, blood lipid and serum adiponectin of primary type 2 diabetes mellitus patients. Taiyuan: Shanxi Medical University; 2008
30 Zheng BB, Wei J, Jiang JD, Zhang RS. The therapeutic effects of combination of simvastatin with berberine on the patients with hyperlipemia. Acta Univ Med Nanjing (Nat Sci) 2009; 29: 1493-1497

31 Ye WP. Clinical efficacy of berberine treatment of diabetes. Mod Hosp 2010; 10: 9-10

32 Zhou YF, Huang SJ. Clinical efficacy of berberine in the treatment of 60 patients with hyperlipidemia. Chin J Clin Rat Drug Use 2011; 4: 76-77

33 O'Brien KA. Alternative perspectives: how Chinese medicine understands hypercholesterolemia. Cholesterol 2010; 2010: 723289

34 Xie W, Zhao Y, Du L. Emerging approaches of traditional Chinese medicine formulas for the treatment of hyperlipidemia. J Ethnopharmacol 2012; 140: 345-367

35 Grundy SM, Cleeman II, Merz NB, Brewer B, Clark LT, Hunninghake DB, Pastemak RC, Smith jr. SC, Stone NJ, National Heart, Lung, and Blood Institute, American College of Cardiology Foundation, American Heart Association. Implications of recent clinical trials for the National Cholesterol Education Program Adult Treatment Panel III guidelines. Circulation 2004; 110: 227-239

36 Hermann $R$, von Richter 0 . Clinical evidence of herbal drugs as perpetrators of pharmacokinetic drug interactions. Planta Med 2012; 78: 1458-1477

37 Guo Y, Pope C, Cheng X, Zhou H, Klaassen CD. Dose-response of berberine on hepatic cytochromes P450 mRNA expression and activities in mice. J Ethnopharmacol 2011; 138: 111-118

38 Guo Y, Chen Y, Tan ZR, Klaassen CD, Zhou HH. Repeated administration of berberine inhibits cytochromes P450 in humans. Eur J Clin Pharmacol 2012; 68: 213-217

39 Schulz KF, Grimes DA. Blinding in randomised trials: hiding who got what. Lancet 2002; 359: 696-700

40 Schulz KF, Grimes DA. Allocation concealment in randomised trials: defending against deciphering. Lancet 2002; 359: 614-618 